\title{
Spatial Attention Does Not Strongly Modulate Neuronal Responses in Early Human Visual Cortex
}

\author{
Daniel Yoshor, ${ }^{1}$ Geoffrey M. Ghose ${ }^{2}$ William H. Bosking, ${ }^{3}$ Ping Sun, ${ }^{1}$ and John H. R. Maunsell ${ }^{4}$ \\ ${ }^{1}$ Department of Neurosurgery, Baylor College of Medicine, Houston, Texas 77030, ${ }^{2}$ Departments of Neuroscience, Radiology, and Psychology, University of \\ Minnesota, Minneapolis, Minnesota 55455, ${ }^{3}$ Department of Neuroscience, University of Texas, Austin, Texas 78712, and ${ }^{4}$ Department of Neurobiology and \\ Howard Hughes Medical Institute, Harvard Medical School, Boston, Massachusetts 02115
}

\begin{abstract}
Attention can dramatically enhance behavioral performance based on a visual stimulus, but the degree to which attention modulates activity in early visual cortex is unclear. Whereas single-unit studies of spatial attention in monkeys have repeatedly revealed relatively modest attentional modulations in V1, human functional magnetic resonance imaging studies demonstrate a large attentional enhancement of the blood oxygen level-dependent (BOLD) signal in V1. To explore this discrepancy, we used intracranial electrodes to directly measure the effect of spatial attention on the responses of neurons near the human occipital pole. We found that spatial attention does not robustly modulate stimulus-driven local field potentials in early human visual cortex, but instead produces modest modulations that are consistent with those seen in monkey neurophysiology experiments. This finding suggests that the neuronal activity that underlies visual attention in humans is similar to that found in other primates and that behavioral state may alter the linear relationship between neuronal activity and BOLD.
\end{abstract}

Key words: attention; vision; spatial; local field potentials; psychophysics; human

\section{Introduction}

How does attention to a spatial location enhance behavioral performance based on a visual stimulus within the attended location (Pashler, 1988)? Does attention predominantly enhance the relatively complex representations found in later stages of visual processing, as suggested by single-unit studies in monkeys, or does it also strongly modulate even the simple visual representations encoded in early visual cortex, as suggested by human functional magnetic resonance imaging (fMRI) studies (Kanwisher and Wojciulik, 2000; Pessoa et al., 2003)? Overall, single-unit (Maunsell and Cook, 2002; Reynolds and Chelazzi, 2004) and local field potential (LFP) (Mehta et al., 2000) recordings in monkeys suggest that attention has relatively modest effects on the activity of the earliest visual areas, with greater modulations in progressively later stages. Until recently, studies in human subjects, particularly scalp event-related potential (ERP) recordings (Hillyard and Anllo-Vento, 1998), were consistent with monkey single-unit data. However, several fMRI studies have challenged this understanding by revealing that spatial attention is associated with a pronounced increase in the visually evoked blood oxygen level-dependent (BOLD) response in V1 (Kanwisher and Wojciulik, 2000; Pessoa et al., 2003). This discrepancy between monkey single-unit and human fMRI data is striking because

\footnotetext{
Received June 28, 2007; revised 0ct. 18, 2007; accepted 0ct. 19, 2007.

This work was supported by grants from the National Institutes of Health (D.Y., J.H.R.M.). J.H.R.M. is an Investigator with the Howard Hughes Medical Institute. We thank L. Rhodes, E. Mizrahi, I. Goldsmith, A. Verma, H. S. Levin, M. Beauchamp, and R. G. Grossman for helpful comments and other assistance.

Correspondence should be addressed to Daniel Yoshor, Department of Neurosurgery, Baylor College of Medicine, One Baylor Plaza, Houston, TX 77030. E-mail: dyoshor@bcm.edu.

DOI:10.1523/JNEUROSCI.2944-07.2007

Copyright $\odot 2007$ Society for Neuroscience $\quad$ 0270-6474/07/2713205-05\$15.00/0
}

many fMRI studies of sensory responses in human brain have confirmed results from single-unit studies in nonhuman primates, and simultaneous measurements of multiunit activity and local field potentials and the BOLD signal in anesthetized monkey visual cortex reveal a strong positive correlation (Logothetis and Wandell, 2004). Although ERP data generally argue against a large attentional effect in human V1, these data are limited by the technique's low spatial resolution. In fact, a study that combined ERP with event-related magnetic field recordings and fMRI suggests that a waveform usually thought to reflect extrastriate activity may actually reflect a robust, albeit late (latency $150-225 \mathrm{~ms}$ ), attentional modulation in human V1 (Noesselt et al., 2002).

To explore this question, we used subdural electrodes anatomically located on early visual cortex in six human subjects (patients undergoing clinical studies to characterize uncontrolled epilepsy) to measure the effect of spatial attention on neuronal activity. Surface electrodes recordings offer an advantage over scalp ERP data because they reflect local activity measured directly from a small region of cortex and can provide small, well defined receptive fields (RFs) (Yoshor et al., 2006). Subjects alternately attended to visual stimuli inside and outside the RF of an electrode positioned on early human visual cortex. We found that spatial attention does not robustly modulate stimulusdriven local field potentials in early human visual cortex, but instead produces modest modulations that are consistent with those seen in monkey neurophysiology experiments.

\section{Materials and Methods}

Subjects. The Baylor College of Medicine Institutional Review Board approved all experimental procedures in this study. Data were collected from eight patients with medically intractable epilepsy who had under- 
gone implantation of subdural electrodes in occipital cortex for clinical purposes and consented to participate in this research study. Patients were screened before surgery, and only those with intact visual fields and normal corrected visual acuity were included. No subject had an MRIidentifiable lesion in the vicinity of the recording electrode used in the experiments.

Recordings. All recording electrodes used in this study were anatomically localized to early visual areas in the occipital lobe [generally V1/V2 as defined by fMRI mapping studies (Sereno et al., 1995; DeYoe et al., 1996; Dougherty et al., 2003)]; the electrode positions were determined by fusing postimplantation computed tomography images with preoperative MR images. These standard clinical subdural electrodes (recording surface $=2.2 \mathrm{~mm}$ diameter; AdTech, Racine, WI) were used to record local field potentials while patients performed the behavioral tasks described below. Experiments took place in a clinical epilepsy-monitoring unit in one or two sessions that occurred between 2 and $4 \mathrm{~d}$ after electrode implantation. Clinical recordings with the implanted electrodes lasted from 5 to $15 \mathrm{~d}$ and continued uninterrupted during experimental sessions.

The local field potentials were amplified and filtered using a portable EEG unit (Nihon Kohden, Foothill Ranch, CA). Voltages from each electrode were typically referenced to a distant electrode, bandpass filtered $(0.3-120 \mathrm{~Hz})$, and sampled at $250 \mathrm{~Hz}$ (16 bit resolution). Using previously described techniques (Yoshor et al., 2006), the spatial RF was mapped for an electrode positioned in early visual cortex in each subject by recording responses to small, high-contrast checkerboard stimuli presented at different locations throughout the central visual field (supplemental Fig. 1, available at www.jneurosci.org as supplemental material). The subsequent experiments were set up and optimized for the spatial RF properties of a single electrode in each subject that was selected because it both anatomically lay on early visual cortex and produced a robust, spatially restricted, and early latency response to simple, highly contrasted checkerboard stimuli that is characteristic of early visual cortex (Yoshor et al., 2006).

Behavioral task. During experimental sessions, subjects were seated in their hospital bed facing a calibrated liquid crystal display video monitor (VP150, 1024 by 768 pixels; Viewsonic, Walnut, CA) at a viewing distance of $57 \mathrm{~cm}$, with a resulting display size of $30.5 \times 22.9^{\circ}$. The primary data for this study were collected while the subject performed a peripheral orientation change detection task while maintaining gaze on a fixation point at screen center (Fig. 1). Eye movements were not monitored. However, because the RFs of these recording electrode in early visual cortex are small and spatially restricted, significant eye movements would have dramatically reduce the evoked response, which in fact did not occur. At the beginning of each trial, a fixation point appeared at screen center followed by single Gabor patch positioned to cue the subject as to which one of the two positions (inside the RF or outside the RF) should be attended in the upcoming block of trials. During each trial, two identical small achromatic Gabor patches $\left(0.5-2\right.$ cycles ${ }^{\circ} ; \sigma, 1.5-3^{\circ}$, with the same average luminance as the gray background) were simultaneously flashed on the screen at a rate of $2-4 \mathrm{~Hz}$ and a duty cycle of $20-50 \%$, with one patch centered on the receptive field of the mapped electrode and the other one in the unresponsive position in the diametrically opposite portion of the contralateral hemifield, well outside the mapped RF. The orientation of one of the two Gabors changed for one presentation ( $1-64^{\circ}$ orientation difference) at a random time $1-6 \mathrm{~s}$ after stimulus onset and then returned to its original orientation for subsequent presentations. Subjects responded to detected orientation changes with a button press, and the allowed response time was $750 \mathrm{~ms}$ after an orientation change occurred. The range of orientation changes and the size and presentation rate of the Gabor patches were varied using on-line analysis to allow data collection at and around each subject's behavioral threshold for detecting orientation changes.

For three of the six subjects, the orientation change occurred more frequently at the cued location (in $71 \%$ of trials), but subjects were instructed to respond to orientation changes in either the cued or uncued location. Data from invalidly cued trials were not used for analysis of neuronal responses, but they provided behavioral measurements to confirm that the subject was selectively attending. The impact of attention on
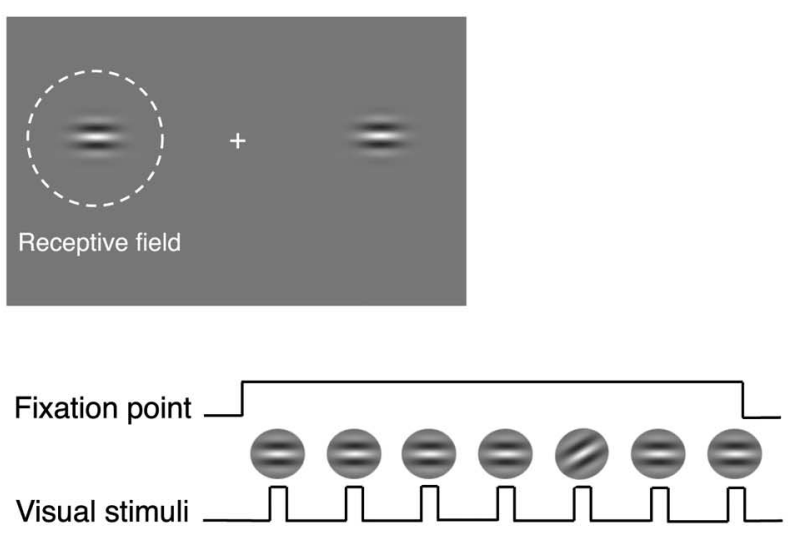

Response period

Time

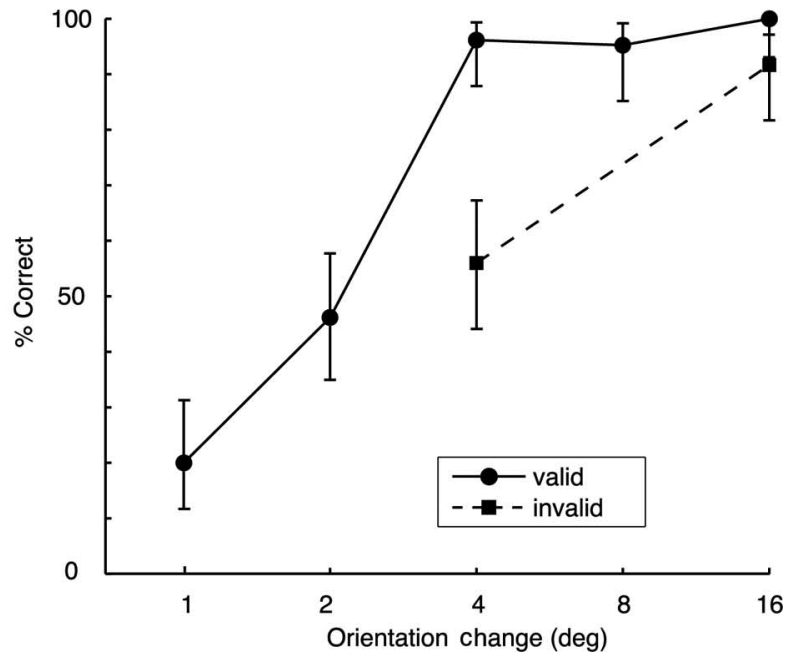

Figure 1. $\quad \boldsymbol{a}$, Spatial attention task. Trials began with the appearance of a fixation cross at screen center, followed by a cue to attend either to a position inside the recording electrode's receptive field (inside the RF) or to an unresponsive position in the diametrically opposite location (outside the RF). During each trial, two identical Gabor patches were simultaneously flashed in both positions and the orientation of one of the two Gabors changed at a random time in one of the two positions. Subjects were instructed to respond to press a button in response to any change in the orientation of the Gabor stimuli. $\boldsymbol{b}$, Effect of spatial attention on behavioral performance for a sample subject. Psychophysical data collected concurrently with recording of neuronal responses illustrates the percentage of correct detections of different orientation changes presented during the spatial attention task. Error bars indicate $68 \%$ binomial confidence intervals. Invalid cues were used only on trials with $4^{\circ}$ or $16^{\circ}$ orientation changes. The significant enhancement of detection of the $4^{\circ}$ orientation change for valid versus invalid cues provides behavioral confirmation that the subject was preferentially directing attention to the cue location.

behavioral performance was measured by comparing the proportion of correct responses for target presentations on the cued side (valid cues) to the proportion of correct responses on the uncued side (invalid cues) using a paired $t$ test $(p<0.05)$. For the other three subjects, the orientation changes only occurred at the cued location. This was explained in advance to these three subjects to enable them to maximally attend to the Gabor stimulus at the cued site, while ignoring the simultaneously appearing identical stimulus at the other location. Although there is no behavioral confirmation of attention for these three subjects, it is likely that the difference in the amount of attention projected to the cued versus the noncued side is even greater because for these subjects, the uncued side did not simply receive less attention, but was ignored. 

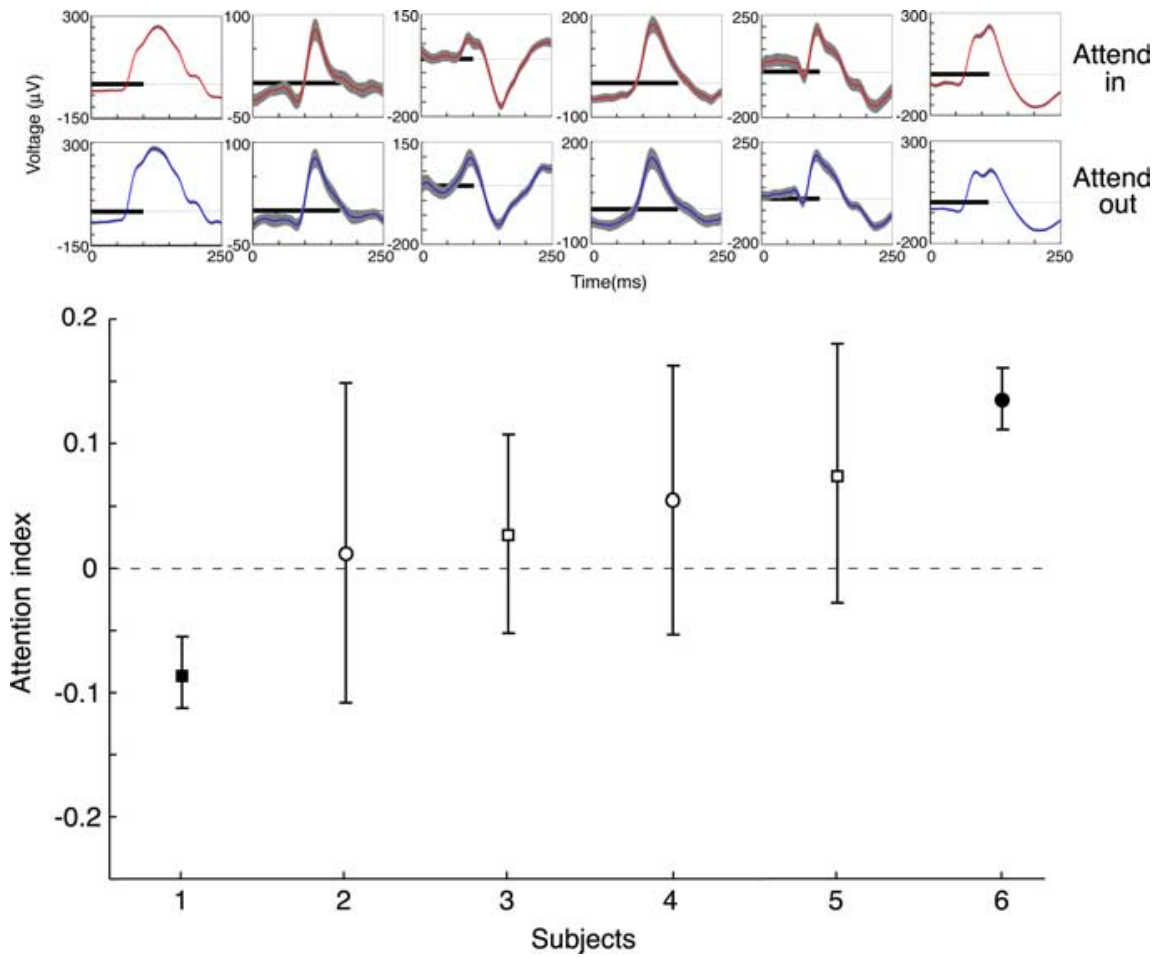

Figure 2. Average LFP responses to a Gabor stimulus with attention directed inside (red traces, top) or outside (blue traces, bottom) the RF demonstrates similar waveforms for both conditions. Gray bands around traces mark the $95 \%$ confidence intervals. The dark horizontal bars that overlie the voltage tracings indicate the period of stimulus presentation. Attentional index (AI) values displayed in the plot at the bottom demonstrate little attentional enhancement of visual responses. Squares indicate subjects with and circles indicate subjects without behavioral verification of attention. Solid symbols indicate subjects with effects that were individually statistically significant. Subject 1 is the subject from Figure $1 b$ and supplemental Figure 1 (available at www.jneurosci.org as supplemental material).

Analysis. Analyses of physiological recordings were performed using custom software generated for Matlab (MathWorks, Natick, MA) as previously described (Yoshor et al., 2006). After excluding excessively noisy ( $>3$ SDs from the mean voltage) or saturated responses, all remaining voltage responses were filtered using a Savitzky-Golay polynomial filter ("sgolayfilt" function in Matlab, with polynomial order set to 5 and frame size set to 11 , where the voltage waveforms were typically composed of 63 samples from a $250 \mathrm{~ms}$ time period from stimulus presentation). The filtered voltage responses for all stimulus presentations from remaining correct trials, beginning with the second stimulus presentation and extending through the last stimulus before the target appeared (and not including the actual target stimulus with the change in orientation and any subsequent stimuli in a trial), were then averaged to obtain the average voltage response for both the "attend inside the RF" and the "attend outside the RF" conditions. The 95\% confidence intervals for each of the averaged voltage responses were determined using a bootstrap procedure. The rms deviation from the mean was then calculated as an index of response strength and used to quantify the ratio between the response strength for the "attend in" and the "attend out" conditions. Tests of statistical differences between the responses to identical stimulus presentations with different levels of attention were done using a $t$ test $(p<0.05)$.

\section{Results}

To measure the effect of spatial attention on field potential responses in early human visual cortex, identical Gabor stimuli were presented in two visual field locations, one inside the RF of a recording electrode located on the occipital pole, and one in a position diametrically opposed across the fixation point (a site that produced no visual response) (supplemental Fig. 1, available at www.jneurosci.org as supplemental material). Subjects fixated on a cross at screen center while the two Gabor stimuli were concurrently flashed at the two specific positions in the display, and they pressed a button when they detected a change in the orientation of a Gabor stimulus at either location (Fig. 1a). Trials were organized into blocks to encourage sustained spatial attention, and before each block the subjects were cued to direct their attention to the spatial location at which the orientation change was more likely to occur, which alternated across blocks between the position inside the RF and the position outside the RF. In this manner, we obtained multiple recordings of neuronal responses in early visual cortex to identical visual stimuli, but with attention directed to different locations.

For three of the six subjects described in this study, the orientation change (the target stimulus for the task) only appeared at the cued location, so the subjects could fully allocate their attention to that location. Although this experimental design offers the advantage of inducing subjects to maximally attend to the cued spatial location, it does not allow for behavioral verification of attention. Therefore, for the remaining three subjects, the majority of the orientation changes were also presented at the cued location, but a small proportion of the orientation changes (29\%) occurred on the uncued side (probe stimuli). This latter design enabled us to measure psychophysical performance in parallel with electrophysiological responses and to obtain simultaneous behavioral verification of attention. Five subjects participated in this latter version of the experiment, and in each subject, performance was better for detection of targets at the cued location, as would be expected if attention were directed preferentially to this location. The subsequent analysis includes only the three subjects whose performance was significantly better for a stimulus in the cued location $\left(\mathrm{X}^{2}, p<0.05\right)$, and who therefore demonstrated clear behavioral verification of attention concurrent with the collection of physiological data.

Figure $1 b$ illustrates the behavioral data collected from a sample subject concurrent with the collection of neuronal responses. The plot shows significantly better performance in detecting a $4^{\circ}$ orientation change when it occurred at the cued location (valid cue), confirming that the subject attended preferentially to the cued location. The other subjects had similar performance. The average for the three subjects tested with invalid cues was $91 \%$ correct for the cued location and $47 \%$ correct for the uncued location for a near-threshold orientation change [hits/(hits + misses)], with a false alarm rate of $<5 \%$.

For all six subjects, attentional modulations of visual responses in early visual cortex were modest (Fig. 2). The mean response waveforms had a similar shape and amplitude regardless of where attention was directed. Figure 2 displays an attentional index (AI) value for each subject, calculated from the average rms voltage values $40-250 \mathrm{~ms}$ after stimulus presentation using the following equation: $\mathrm{AI}=\left(\mathrm{rms}_{\text {attend in }}-\mathrm{rms}_{\text {attend out }}\right) /$ $\left(\mathrm{rms}_{\text {attend in }}+\mathrm{rms}_{\text {attend out }}\right)$. The mean AI for the six subjects was 
0.04 (SD, 0.07 ), which corresponds to an $8 \%$ increase in visually driven local cortical response. This mean change was not statistically significant ( $p=0.93, t$ test). The attentional effect varied considerably between subjects; however, this is expected, because similar or greater variance is seen in the effects of attention on single-unit and LFP responses in monkey visual cortex as well as in BOLD data from human visual cortex (Huk and Heeger, 2000). The range of effects seen here is comparable with attentional modulation of LFP in monkey V1 [0.03; SD, 0.04 (Mehta et al., 2000)]. For two subjects, the attentional modulation was individually statistically significant (filled symbols), including, in one case, a decreased response with increased spatial attention, as is sometimes seen with single-unit recordings (Maunsell and Cook, 2002). Overall, these results suggest that spatial attention affects neuronal responses in early human visual cortex to an extent that is comparable with that seen in electrophysiological studies in nonhuman primates.

\section{Discussion}

Our results, unlike those derived from human fMRI experiments, suggest that spatial attention does not strongly modulate visual responses in early human visual cortex. Our task paradigm and experimental approach was based on electrophysiological studies of attention in nonhuman primates, in which stimulation parameters and timing are based on measured receptive fields properties (Haenny et al., 1988; Spitzer et al., 1988; Motter, 1993; Luck et al., 1997; McAdams and Maunsell, 2000). Because fMRI techniques are incapable of such spatial and temporal resolution, the majority of such studies measure responses over many seconds and employ stimuli that are far more spatially extensive. However, there are several factors that make it unlikely that task or stimulus differences are responsible for the discrepancy between our results and fMRI studies of attention in early visual areas. First, significant spatial attentional effects have been reported using fMRI with a wide variety of task requirements, including pop-out detection (Martinez et al., 1999), speed discrimination (Gandhi et al., 1999), contrast detection (Ress et al., 2000), feature conjunction (Brefczynski and DeYoe, 1999), luminance discrimination (O'Connor et al., 2002), and directional discrimination (Somers et al., 1999). Second, fMRI studies have found robust attentional effects in early visual cortex using a variety of stimuli, including symbol arrays (Martinez et al., 1999), sinusoid gratings (Gandhi et al., 1999), angular wedges (O'Connor et al., 2002), and radially symmetric annuli (Brefczynski and DeYoe, 1999; Somers et al., 1999; Ress et al., 2000). In this regard, two studies are particularly notable: Gandhi et al. (1999) used stimuli (sinusoidal gratings that were $3^{\circ}$ in diameter and at an eccentricity of $7^{\circ}$ ) that were very similar to the stimuli we used, and Ress et al. (2000) reported significant modulation in the absence of any visual stimulation. Given this range of stimuli, it seems unlikely that our results are strongly constrained by our choice of visual stimuli, which were chosen to optimize visual responses at our recording sites. Third, most of the fMRI studies have cycled between attended locations every $20-40 \mathrm{~s}$, which is not considerably different from the amount of time spent within a trial block in our design. Finally, although it is true that neuronal responses may not be modulated by attention if subjects are presented with a task that is not challenging (Boudreau et al., 2006), we do not think that our task was too easy, because it required nearthreshold discriminations, behavioral measures showed that attention was critical for achieving good performance (Fig. 1b), and the task performance in our study was similar to that reported in fMRI studies.
It is not the case that our LFP recordings are unable to reveal pronounced attentional modulation. In contrast to the current results, we have observed robust attentional modulations when recording visual responses in the basal or inferior temporal lobe, a late stage in the ventral stream characterized by large receptive fields and object-selective responses. The large receptive fields at these sites, as opposed to the small and spatially restricted fields in early visual areas, prevent us from performing a spatial attention experiment for these locations. However, we have observed strong enhancements of visual responses in this region when a stimulus is a target, as opposed to a distractor, during a visual search task (supplemental Fig. 2, available at www.jneurosci.org as supplemental material).

The finding that local field potentials recorded directly from human occipital cortex are not markedly enhanced by spatial attention is consistent with single-unit physiological studies performed in monkeys (Maunsell and Cook, 2002; Reynolds and Chelazzi, 2004). Our human data and monkey single-unit data differ from the robust attentional effect seen with fMRI [for review, see Kanwisher and Wojciulik (2000) and Pessoa et al. (2003)], which reveals attentional modulations of visual responses in V1 of 25-100\% (Gandhi et al., 1999; Somers et al., 1999), and from the large delayed attentional effect in V1 suggested by a recent human study combining fMRI and ERP (Noesselt et al., 2002).

The current data argue against several possible explanations for the discrepancy between the small modulations seen in singleunit studies and the more sizable effects seen with BOLD. Because our recordings were from naive humans, the difference is unlikely to reflect a fundamental difference between human and monkey neurophysiology or a reduction in attentional modulation in monkeys caused by the extensive training they typically receive. Because our measurements were based on local field potential recorded from relatively large surface electrodes $(2.2 \mathrm{~mm}$ diameter), the difference probably does not reflect the effects of combining signals from small versus large neuronal populations.

The explanation that is the most consistent with our results is that the large BOLD modulations seen in human V1 reflect hemodynamic changes that are not associated with modulations of single-unit or local field potential activity. This is supported by the recent finding that spatial attention adds a constant BOLD signal independent of stimulus strength in V1 (Buracas and Boynton, 2007). Because stimulus onsets attract attention, such a hemodynamic effect might explain the temporal nonlinearities observed in most fMRI studies, in which responses to brief auditory and visual stimuli are much larger than expected from the responses to stimuli of longer durations (Birn et al., 2001). Thus, behavioral state may alter the linear relationship between activity and BOLD that has been suggested by V1 experiments (Boynton et al., 1996) and is the basis for many fMRI studies that use linear methods to infer neural activity (Friston et al., 1994). In particular, our study suggests that large modulations of BOLD signals may not be associated with large modulations of single-unit or local field potential activity in primary visual cortex.

\section{References}

Birn RM, Saad ZS, Bandettini PA (2001) Spatial heterogeneity of the nonlinear dynamics in the FMRI BOLD response. NeuroImage 14:817-826.

Boynton GM, Engel SA, Glover GH, Heeger DJ (1996) Linear systems analysis of functional magnetic resonance imaging in human V1. J Neurosci 16:4207-4221

Boudreau CE, Williford TH, Maunsell JH (2006) Effects of task difficulty and target likelihood in area V4 of macaque monkeys. J Neurophysiol 96:2377-2387. 
Brefczynski JA, DeYoe EA (1999) A physiological correlate of the 'spotlight' of visual attention. Nat Neurosci 2:370-374.

Buracas GT, Boynton GM (2007) The effect of spatial attention on contrast response functions in human visual cortex. J Neurosci 27:93-97.

DeYoe EA, Carman GJ, Bandettini P, Glickman S, Wieser J, Cox R, Miller D, Neitz J (1996) Mapping striate and extrastriate visual areas in human cerebral cortex. Proc Natl Acad Sci USA 93:2382-2386.

Dougherty RF, Koch VM, Brewer AA, Fischer B, Modersitzki J, Wandell BA (2003) Visual field representations and locations of visual areas V1/2/3 in human visual cortex. J Vis 3:586-598.

Friston KJ, Jezzard P, Turner R (1994) Analysis of functional MRI timeseries. Hum Brain Mapp 1:153-171.

Gandhi SP, Heeger DJ, Boynton GM (1999) Spatial attention affects brain activity in human primary visual cortex. Proc Natl Acad Sci USA 96:3314-3319.

Haenny PE, Maunsell JH, Schiller PH (1988) State dependent activity in monkey visual cortex. II. Retinal and extraretinal factors in V4. Exp Brain Res 69:245-259.

Hillyard SA, Anllo-Vento L (1998) Event-related brain potentials in the study of visual selective attention. Proc Natl Acad Sci USA 95:781-787.

Huk AC, Heeger DJ (2000) Task-related modulation of visual cortex. J Neurophysiol 83:3525-3536.

Kanwisher N, Wojciulik E (2000) Visual attention: insights from brain imaging. Nat Rev Neurosci 1:91-100.

Logothetis NK, Wandell BA (2004) Interpreting the BOLD signal. Annu Rev Physiol 66:735-769.

Luck SJ, Chelazzi L, Hillyard SA, Desimone R (1997) Neural mechanisms of spatial selective attention in areas V1, V2, and V4 of macaque visual cortex. J Neurophysiol 77:24-42.

Martinez A, Anllo-Vento L, Sereno MI, Frank LR, Buxton RB, Dubowitz DJ, Wong EC, Hinrichs H, Heinze HJ, Hillyard SA (1999) Involvement of striate and extrastriate visual cortical areas in spatial attention. Nat Neurosci 2:364-369.

Maunsell JH, Cook EP (2002) The role of attention in visual processing. Philos Trans R Soc Lond B Biol Sci 357:1063-1072.
McAdams CJ, Maunsell JH (2000) Attention to both space and feature modulates neuronal responses in macaque area V4. J Neurophysiol 83:1751-1755.

Mehta AD, Ulbert I, Schroeder CE (2000) Intermodal selective attention in monkeys. I: distribution and timing of effects across visual areas. Cereb Cortex 10:343-358.

Motter BC (1993) Focal attention produces spatially selective processing in visual cortical areas V1, V2, and V4 in the presence of competing stimuli. J Neurophysiol 70:909-919.

Noesselt T, Hillyard SA, Woldorff MG, Schoenfeld A, Hagner T, Jancke L, Tempelmann C, Hinrichs H, Heinze HJ (2002) Delayed striate cortical activation during spatial attention. Neuron 35:575-587.

O’Connor DH, Fukui MM, Pinsk MA, Kastner S (2002) Attention modulates responses in the human lateral geniculate nucleus. Nat Neurosci 5:1203-1209.

Pashler HE (1988) The psychology of attention. Cambridge, MA: MIT.

Pessoa L, Kastner S, Ungerleider LG (2003) Neuroimaging studies of attention: from modulation of sensory processing to top-down control. J Neurosci 23:3990-3998.

Ress D, Backus BT, Heeger DJ (2000) Activity in primary visual cortex predicts performance in a visual detection task. Nat Neurosci 3:940-945.

Reynolds JH, Chelazzi L (2004) Attentional modulation of visual processing. Annu Rev Neurosci 27:611-647.

Sereno MI, Dale AM, Reppas JB, Kwong KK, Belliveau JW, Brady TJ, Rosen BR, Tootell RB (1995) Borders of multiple visual areas in humans revealed by functional magnetic resonance imaging. Science 268:889-893.

Somers DC, Dale AM, Seiffert AE, Tootell RB (1999) Functional MRI reveals spatially specific attentional modulation in human primary visual cortex. Proc Natl Acad Sci USA 96:1663-1668.

Spitzer H, Desimone R, Moran J (1988) Increased attention enhances both behavioral and neuronal performance. Science 240:338-340.

Yoshor D, Bosking WH, Ghose GM, Maunsell JH (2007) Receptive fields in human visual cortex mapped with surface electrodes. Cereb Cortex 17: 2293-2302. 